Dans le dossier SwissDRG, I'enthousiasme des partenaires faiblit quand il s'agit de passer à une phase de concrétisation. Ce phénomène est particulièrement évident en ce qui concerne la recherche concomitante: les responsabilités se diluent et rien de crédible ne démarre dans un délai raisonnable. Dans son éditorial, B. Meyer nous fait part des conséquences que la FMH tire de ce constat, ainsi que des premières mesures qu'elle entend prendre pour établir un projet de recherche sérieux au profit de nos membres concernés.
Dans un autre registre, le travail de G. Lang et coll., également présent dans ce numéro (p. 1265), remet très à propos le doigt sur la protection des données à l'ère de SwissDRG. L'article suggère une solution pragmatique, praticable et juridiquement conforme à ce dilemme persistant.

Dr Pierre-François Cuénoud Membre du Comité Central de la FMH Responsable du domaine SwissDRG

\title{
SwissDRG et recherche concomitante: qui osera le premier pas?
}

Très tôt, la FMH a relevé l'importance d'une recherche concomitante dans la perspective de l'introduction du système des forfaits par cas SwissDRG dans tous les hôpitaux suisses. Où en sommes-nous aujourd'hui, 16 mois avant cette introduction?

Résumons: il y a un an, la FMH a présenté à SwissDRG SA un concept précis pour la recherche concomitante à l'occasion de l'introduction de SwissDRG. Depuis lors, un temps précieux s'est écoulé sans que le coup d'envoi n'ait été donné pour la mise en œuvre de cette recherche. Il faut pré-

\section{Détecter à temps les imperfections -}

\section{la FMH a mis au concours une étude concomitante}

ciser que SwissDRG SA a décidé qu'elle ne voulait pas en assumer la responsabilité et qu'elle préfère se borner à élaborer un instrument pour le contrôle des coûts et des prestations. L'assurance-qualité a finalement été confiée à l'Association nationale pour le développement de la qualité dans les hôpitaux et les cliniques (ANQ). L'OFSP, quant à lui, a mis ce temps à profit pour élaborer une étude de faisabilité destinée à évaluer la révision de la LAMal. Mais à notre grand regret, les études prévues dans le cadre de cette évaluation ne démarreront qu'après l'introduction du système SwissDRG. En outre, il est d'ores et déjà évident que l'OFSP n'examinera pas divers points importants pour le corps médical tels que l'évolution de la relation médecin-patient ou la charge administrative dans les hôpitaux.

Devrons-nous subir le même sort que nos collègues allemands? Nous le craignons. En Allemagne, au printemps 2010, c'est-à-dire six ans après l'introduction du système G-DRG, les premiers résultats de la recherche concomitante ont été publiés dans un rapport comptant 895 pages. Le bilan tiré par la Chambre médicale allemande est décevant: précisément les questions importantes concernant la prise en charge des patients et le corps médical sont restées sans réponse. Il n'est ainsi guère possible de détecter et de corriger les imperfections du système.

Pour épargner une déception du même ordre aux médecins suisses, la FMH a décidé d'examiner elle-même les points les plus importants pour le corps médical. Ces questions, ignorées par l'OFSP et les autres partenaires de la santé mais considérées - à juste titre - comme particulièrement importantes par nos sociétés de discipline et nos organisations faîtières, touchent directement le corps médical: les médecins hospitaliers ont-il encore suffisamment de temps pour leurs patients? Le travail administratif s'accroît-il? Comment évoluent la relation médecin-patient et la collaboration avec les autres spécialistes de la santé? Le médecin peut-il encore prodiguer le traitement qu'il estime approprié pour son patient à l'hôpital? Le personnel est-il suffisant pour offrir un traitement de qualité? Qu'en est-il de l'état de santé des médecins eux-mêmes face aux nombreuses exigences?

Pour pouvoir répondre à ces questions et à d'autres, la FMH a mis au concours une étude scientifique (cf. www. fmh.ch $\rightarrow$ Tarifs $\rightarrow$ SwissDRG $\rightarrow$ Recherche concomitante). Ses résultats en main, le corps médical disposera d'un instrument étayé de chiffres et de faits pour repérer suffisamment tôt les développements inadéquats, en vue de sensibiliser le monde politique à ce sujet.

Il est évident que cette étude n'est qu'un premier pas pour répondre aux questions brûlantes que se pose le corps médical en lien avec les changements en cours dans les hôpitaux. D'autres démarches réunissant les efforts des partenaires de la santé sont nécessaires pour atteindre l'objectif d'un monitorage à large échelle en vue d'une politique durable de la santé.

Beatrix Meyer, Cheffe du Service tarifaire de la FMH et du domaine SwissDRG 